\title{
Structure-From-Motion and RGBD Depth Fusion
}

\author{
Akash Chandrashekar, John Papadakis, Andrew Willis \\ Department of Electrical and Computer Engineering \\ University of North Carolina at Charlotte \\ Charlotte, NC 28223 \\ Email: achandr9@uncc.edu
}

\author{
Jamie Gantert \\ Munitions Directorate \\ Air Force Research Laboratory \\ Eglin AFB, Florida 32542 \\ Email: jamie.gantert.1@us.af.mil
}

\begin{abstract}
This article describes a technique to augment a typical RGBD sensor by integrating depth estimates obtained via Structure-from-Motion (SfM) with sensor depth measurements. Limitations in the RGBD depth sensing technology prevent capturing depth measurements in four important contexts: (1) distant surfaces $(>5 \mathrm{~m}),(2)$ dark surfaces, (3) brightly lit indoor scenes and (4) sunlit outdoor scenes. SfM technology computes depth via multi-view reconstruction from the RGB image sequence alone. As such, SfM depth estimates do not suffer the same limitations and may be computed in all four of the previously listed circumstances. This work describes a novel fusion of RGBD depth data and SfM-estimated depths to generate an improved depth stream that may be processed by one of many important downstream applications such as robotic localization and mapping, as well as object recognition and tracking.
\end{abstract}

\section{INTRODUCTION}

RGBD sensors are a relatively new class of image sensors. Their key novel feature is the ability to simultaneously capture color "RGB" images of the scene and depth "D" images of scene- hence the term "RGBD." RGB images are captured using a conventional visible light camera that incorporates a lens to focus light rays from scene locations onto distinct light-sensitive pixels of image sensor. Structured light RGBD sensors consist of three integrated devices: an infrared (IR) projector, an IR camera and an RGB camera in a rigid relative geometry to create a single sensor that captures color-attributed $(X, Y, Z)$ surface data at ranges up to $\sim 6 \mathrm{~m}$ with frame rates up to $30 \mathrm{~Hz}$. RGBD sensors have a wide range of applications which include mapping, localization, pose estimation, and object recognition. They have become popular for their easeof-use and low cost in comparison with other visual sensor technologies such as LIDAR, and have been incorporated into consumer products like mobile phones, gaming consoles, and automobiles [1].

\section{A. RGBD Sensing Technology and Limitations}

Depth image formation is accomplished using structured light technology to measure the geometric position of viewed surfaces. This is accomplished by illuminating scene surfaces with an infrared (IR) projector having a known pattern and then using an IR camera to capture the projected pattern [2]. Deformation of the projected pattern over scene object is analyzed and used to triangulate the depth of scene surfaces with respect to the camera's optical axis. The IR projector operates outside of visible light frequencies and, as such, does not interfere with the captured RGB stream pixel values.
Despite the popularity of RGBD sensors, their utility for generic depth measurement is limited in several ways due to shortcomings associated with structured light depth estimation. One significant shortcoming is that RGBD sensors often fail to provide meaningful depth values in sunlit outdoor scenes. Here the infrared radiation from sunlight interferes with the projected pattern, causing the depth estimation process to fail. This phenomenon also occurs in sunlit indoor scenes. RGBD sensors also fail to collect measurements from surfaces having specific reflectance properties. This includes the following three reflectance contexts: (1) "dark" surfaces, i.e., surfaces having a low reflectance, (2) specular, i.e., mirror-like, surfaces and (3) transparent surfaces [3][4]. All of these cases represent instances where the projected IR pattern reflected into the IR camera is not observable from the background due to signal interference, e.g., sunlight, or weak/dim images, e.g., low reflectance, mirror-like and transparent surfaces.

\section{B. Structure from Motion}

The Structure from Motion (SfM) algorithm leverages ideas originally drawn from photogrammetry to estimate the threedimensional structure of a scene from a time series of RGB images from a moving single camera. This is achieved by calibrating the camera [5] to develop a highly-accurate model to describe how 3D positions are projected into camera images. Using this image formation model, the SfM algorithm matches together pixels in separate images that correspond to projections of the same 3D location as the camera moves in the scene. Using the camera projection model and the assumption that matched pixels are measurements of the same 3D world coordinates, the SfM algorithm solves for both the pose of the camera within the global coordinate system and the set of 3D surface positions provided by corresponding image pixels [6]. The SfM problem is non-linear in the unknowns and is typically solved in a two-stage sequence. Stage 1 solves for the relative pose of the camera at the instant the images were recorded. Stage 2 conditions on the estimated camera pose values and solves for the 3D scene structure. Both stages use correspondences between pixels from different images to solve the non-linear equations in the unknown variables. The camera pose tracking problem of Stage 1 is often solved by finding a map that transforms pixels from the original $(x, y)$ coordinate field to new coordinate positions $\left(x^{\prime}, y^{\prime}\right)$ such that both locations correspond to images of the same 3D scene point. The multi-view 3D surface reconstruction of Stage 2 is often solved using the bundle adjustment algorithm [7]. 


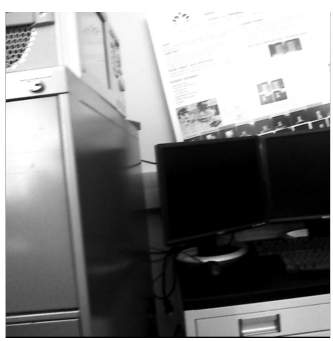

(a)

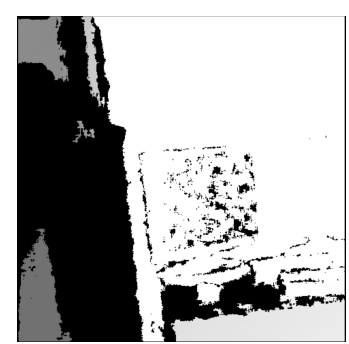

(b)

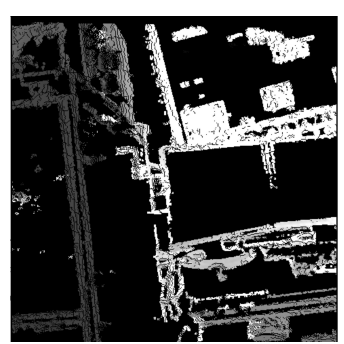

(c)

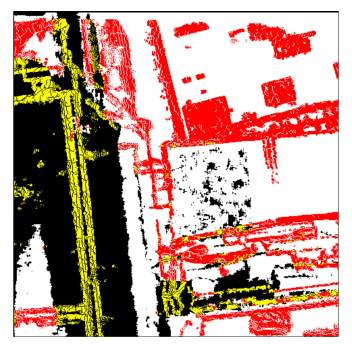

(d)

Figure 1: An overview of the proposed RGBD and SfM fusion algorithm (a) shows a grayscale image of the scene (b) shows the sensed RGBD depth image (c) shows the SfM-estimated depth image and (d) shows the fused image. The fused image has been color-coded as follows: (white) denotes depth locations sensed only by the RGBD sensor, (yellow) denotes depth locations only sensed via SfM, (red) denotes fused (RGBD+SfM) depth locations and (black) denotes depth locations without RGBD or SfM measurements.

While scene reconstruction via SfM produces depth images in contexts where depth cameras fail, this modality for depth estimation also has several shortcomings. Specifically, the theoretical formulation of the SfM problem shows that the scale of the estimated 3D structure cannot be known without prior or outside information. This complicates both the mathematical and computational SfM solutions. SfM also presumes that viewed surfaces are static, i.e., they do not move, and when this assumption is violated reconstructed surfaces are highly inaccurate.

\section{Contribution}

This article seeks to leverage the strengths of structured light sensor derived and SfM derived depth measurements by fusing these measurements into an improved depth image that provides depth measurements in contexts where at least one of the two depth estimation approaches succeeds. Figure 1 shows an RGBD-SfM fusion result for an indoor scene and how the fusion result (Figure 11(d)) captures more scene geometry than either approach independently. Our proposed method to fuse RGBD and SfM depth imagery includes consideration of the RGBD sensor depth noise model, the SfM algorithm depth noise model and also copes with the inherent unknown scale and scale-drift problems intrinsic to SfM. To our knowledge these technical issues have not been discussed elsewhere in the literature.

\section{RELATED WORK AND BACKGROUND INFORMATION}

This article proposes fusion of SfM-estimated depths with depths captured from an RGBD image sensor. This section is dedicated to discussing the relevant aspects of the SfM algorithm and the sensed RGBD measurements necessary to explain the proposed fusion method. Specifically, this section reviews the theoretical details of the SfM algorithm, methods for processing depth images including computing depth images for arbitrary camera poses, and details existing knowledge regarding the sensor measurement noise for RGBD depth measurements.

\section{A. SfM Depth Reconstruction}

The SfM algorithm uses a time sequence of images from a moving camera to recover the 3D geometry of objects viewed by the camera. While this problem can be solved without a calibrated camera, reconstruction accuracy will adversely affected. This work assumes that the camera calibration [8] parameters are known. The SfM algorithm can be broken down into two key steps:

1) Estimation of the camera pose, i.e., position and orientation, at the time each image was recorded,

2) Estimation of the $3 D$ structure of the scene.

As previously mentioned, typical SfM systems solve (1) by computing a map that associates pixels from the original $(x, y)$ coordinate field to new coordinate positions $\left(x^{\prime}, y^{\prime}\right)$ such that both locations correspond to images of the same 3D scene point and (2) via multi-view 3D surface reconstruction algorithm, e.g., bundle adjustment [7]. In the following sections we provide an overview of aspects of the SfM algorithm necessary for the development of the proposed RGBD-SfM depth fusion algorithm.

1) Solving for Image Pixel Correspondences: There are generically two different approaches for finding corresponding observations of the same 3D surface location in multiple images referred to as direct and indirect [9]. In this discussion, we refer to the correspondence problem as a source-to-target matching problem. Let $\mathbf{I}_{t}(x, y)$ denote an image recorded at time $t$ and $\mathbf{I}_{t+\Delta t}(x, y)$ denote a subsequent image measured at time $t+\Delta t$. The correspondence problem seeks to find a map that transforms pixels from the original $(x, y)$ coordinate field of $\mathbf{I}_{t}$ to new coordinate positions $\left(x^{\prime}, y^{\prime}\right)$ in $\mathbf{I}_{t+\Delta t}$ such that $\mathbf{I}_{t}(x, y)$ and $\mathbf{I}_{t+\Delta t}\left(x^{\prime}, y^{\prime}\right)$ correspond to projections of the same 3D scene point.

Indirect Methods: Indirect methods compute this mapping by detecting special $(x, y)$ locations referred to as feature locations with a purpose-built feature detection algorithm, e.g., the Harris corner detector [10]. A description of the image patch in the vicinity of each detected $(x, y)$ location is computed using some feature descriptor algorithm, e.g., Lowe's SIFT descriptor [11]. Feature descriptors seek to provide a vector of values from the image patch data that is invariant to the image 
variations that occur during camera motion. These include but are not limited to the following effects: illumination variation, affine and/or projective invariance, photometric invariance (brightness constancy), and scale invariance.

Popular feature descriptors often prioritize scale and affine invariance as their strengths. The invariance property allows for correspondences to be computed by finding the mapping from the feature descriptor set calculated from image $\mathbf{I}_{t}(x, y)$ to the feature descriptor set calculated from image $\mathbf{I}_{t+\Delta t}(x, y)$. Solutions often assume the map solution is 1-to-1, i.e., a single 3D location can only project to one location in each image, and the descriptor values are invariant across the image pair. As such, solving for the correspondence reduces to an assignment problem where we seek to find the correspondence that minimizes the difference between corresponding descriptors. Let $\mathbf{d}_{i, t}$ denote the $i^{t h}$ descriptor from $\mathbf{I}_{t}(x, y)$ and $\mathbf{d}_{j, t+1}$ denote the $j^{t h}$ descriptor from $\mathbf{I}_{t+\Delta t}(x, y)$. We then specify the indirect correspondence problem as a search for the correspondence set $\mathcal{C}$ consisting of index pairs $(i, j)$ that minimize the total descriptor error as shown in equation (1) with the standard squared vector norm error function shown in equation (2).

$$
\begin{gathered}
\widehat{\mathcal{C}}=\min \sum_{(i, j) \in \mathcal{C}} e\left(\mathbf{d}_{i, t}, \mathbf{d}_{j, t+1}\right), \\
e\left(\mathbf{d}_{i, t}, \mathbf{d}_{j, t+1}\right)=\left\|\mathbf{d}_{i, t}-\mathbf{d}_{j, t+1}\right\|^{2}
\end{gathered}
$$

Direct Methods: Direct methods on the other hand typically iteratively solve for a set of transformation parameters that best align a pair of images by the minimization of pixel-wise errors. An image warping function, $\omega(\mathbf{x}, \xi)$, maps a pixel location, $\mathbf{x}=[x, y]^{t}$, in the original coordinate field to new coordinate positions, $\mathbf{x}^{\prime}$, such that both locations correspond to images of the same 3D scene point. A classical solution to this problem is given by the Lucas-Kanade-Tomassi (LKT) camera tracking algorithm [12].

This is analogous to the process of image alignment, whose goal is to minimize the sum of squared error between two images, the template image $\mathbf{T}(\mathbf{x})$, and the moving image, $\mathbf{I}(\mathbf{x})$. In this method the moving image is mapped onto the coordinate frame of the template image using a warp function, $\omega(\mathbf{x}, \xi)$, as in equation 3 .

$$
\widehat{\xi}=\min _{\xi} \sum_{\mathbf{x}}(\mathbf{I}(\omega(\mathbf{x}, \xi))-\mathbf{T}(\mathbf{x}))^{2}
$$

In equation (3), the warp function, $\omega(\mathbf{x}, \xi)$, maps pixel locations, $\mathbf{x}$, in the template to pixel locations in the image $\mathbf{I}(\mathbf{x})$ using the warp transformation parameters $\xi$. For direct correspondence estimation, $\xi$ is a pose transformation of the viewing camera represented as a unknown $6 \times 1$ vector. We then seek the camera pose transformation parameters, $\widehat{\xi}$, that minimize equation (3) which provides the camera pose change that best explain the differences in these two of images of the same scene. Given two images and the camera pose change between them, one can take the information in one image, and through the warp function map these values into the viewpoint of the other image to establish a correspondence.
In this case the theoretical difference between expected and observed values for the image pair is zero if the camera pose change is known exactly and sensor noise and other outside influences are ignored.

2) Solving for Scene Geometry: There are generally two different approaches for $3 \mathrm{D}$ reconstruction referred to as sparse and dense approaches. Sparse methods reconstruct the 3D scene geometry only for a select subset of the entire image data [13]. This subset is often corner locations or locations marked by some type of extracted feature, e.g., SIFT or SURF. [11], [14] This results in a sparse description of the 3D scene in terms of a sparse cloud of 3D points. In contrast, dense methods [15] reconstruct as many $3 \mathrm{D}$ geometric locations as possible and seek to provide a more complete description of the scene.

Sparse reconstructions often benefit from having a lower computational cost but provide few 3D measurements. Dense reconstructions have higher computational cost but provide a much more complete description of the 3D scene. Dense reconstruction techniques have seen much recent interest, although a highly accurate, dense, and real-time SfM approach has remained elusive.

A third class of algorithms, referred to as semi-dense algorithms [6], seek to strike a compromise between the sparse and dense methods. The reconstruction techniques used are most similar to dense methods, however, only a subset of all image pixels are reconstructed. These approaches leverage the high accuracy of dense reconstruction techniques, but are sparse enough to allow for real-time operation.

Here, reconstruction is limited to those pixels which possess high intensity gradient values. These regions often correspond to scene geometries such as edges, corners, and curves and to other areas of the scene that are highly textured. The thought here is that regions of the image that possess large changes in intensity convey more information than regions that possess less, thus semi-dense reconstructions provide a compressed version of the total dense scene structure.

3) LSD SLAM: We leverage the SfM algorithm proposed by Engel et al. dubbed LSD-SLAM. [16] This approach is a semidense, direct method which optimizes the geometry directly on the image intensities. LSD-SLAM provides as output a reconstruction of the observed 3D environment as a pose-graph of specially designated RGB images called "keyframes" with associated semi-dense depth images. Direct correspondences are found between every RGB frame and each RGB keyframe to estimate the keyframe-to-RGB-frame relative camera pose. This is achieved by Levenberg-Marquardt optimization of the photometric reprojection error between the RGB image pair.

$$
E(\xi)=\sum_{i}\left(I_{r e f}\left(P_{i}\right)-I\left(\omega\left(p_{i}, D_{r e f}\left(p_{i}\right), \xi\right)\right)\right)^{2}
$$

Equation (4) shows the specific image alignment objective function and formalizes the form for equation (3). Here, the warp function relies on the current estimate of depth, $D_{r e f}$, to determine the relative pose change as a similarity transform: $\xi \in \operatorname{sim}(3)$. The reference depth, $D_{\text {ref }}$, is the depth associated with current keyframe, these depth values could be initialized 


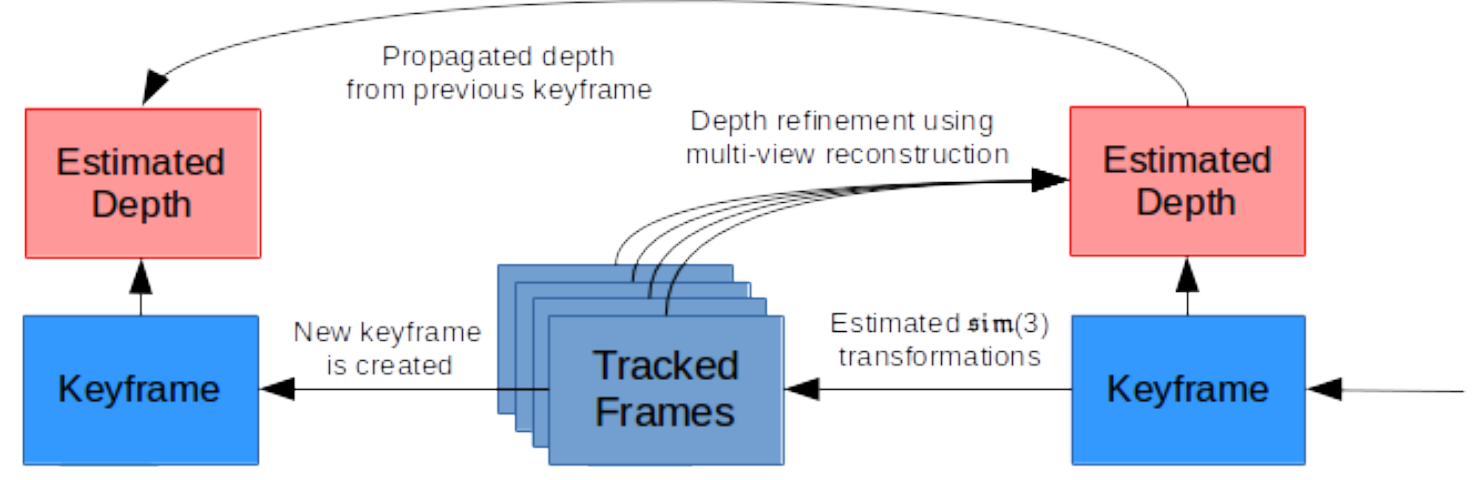

Figure 2: An overview of the LSD SLAM algorithm. Each incoming frame is tracked against the current keyframe. If it does not satisfy the critera for new keyframe creation, it is used along with previous tracked frames for refinement of the estimated depth values of the keyframe. Otherwise, the frame is considered a new keyframe and depth estimates from the previous keyframe are propagated and used for initialization of the new depth estimates.

either with random values or with the depth measurements from a RGBD sensor to initiate the process. For every new frame tracked, the depths associated with the keyframe are continuously refined by performing adaptive baseline stereo 3D reconstruction [6] using the newly tracked frame along with a stack of frames that have been tracked previously. When there is drastic pose change between the tracked frame and keyframe, the current tracked frame is promoted to a keyframe and the depth map from the previous keyframe is propagated to the new keyframe using the estimated pose change and regularized. All keyframes are added as nodes to a pose graph that stores the relative pose between the keyframes as edges/constraints [17]. The pose graph stores the global trajectory of the camera in the 3D scene and a graph optimization algorithm processes the pose graph to improve the camera pose estimates as new image correspondences are found by image matching and loop closures.

\section{B. Depth Image Processing}

Depth fusion requires a significant amount of depth image processing. This section details how depth images can be used to reconstruct 3D point clouds and how point cloud reprojection can be used to predict the depth image captured from a camera in an arbitrary target pose from a measured depth image and knowledge of the relative pose between the the measured image and the target pose.

1) Point Cloud Reconstruction: Measured 3D $(X, Y, Z)$ positions of sensed surfaces can be directly computed from the intrinsic camera parameters and depth image values. Here, the $Z$ coordinate is directly taken as the depth value and the 3D $(X, Y)$ coordinates are computed using the pinhole camera model. In a typical pinhole camera model 3D $(X, Y, Z)$ points are projected to $(x, y)$ image locations [18], e.g., for the image columns the $x$ image coordinate is $x=f_{x} \frac{X}{Z}+c_{x}-\delta_{x}$. However, for a depth image, this equation is re-organized to "back-project" the depth into the 3D scene and recover the 3D $(X, Y)$ coordinates as shown by equation 5 .

$$
\begin{array}{ccc}
X & = & \left(x+\delta_{x}-c_{x}\right) Z / f_{x} \\
Y & = & \left(y+\delta_{y}-c_{y}\right) Z / f_{y} \\
Z & = & Z
\end{array}
$$

where $Z$ denotes the sensed depth at image position $(x, y)$, $\left(f_{x}, f_{y}\right)$ denotes the camera focal length (in pixels), $\left(c_{x}, c_{x}\right)$ denotes the pixel coordinate of the image center, i.e., the principal point, and $\left(\delta_{x}, \delta_{y}\right)$ denote adjustments of the projected pixel coordinate to correct for camera lens distortion.

2) Point Cloud Re-Projection: Depth images can be simulated for camera sensor in arbitrary poses by applying backprojection, transformation on the resulting points, and projection into the new coordinate frame. For discussion, assume that depth image $\mathbf{d}(x, y)$ has been recorded in the "standard" camera/optical coordinate system where the origin corresponds to the camera focal point, the $z$-axis corresponds to the depth/optical axis extending out into the viewed scene, the $x$-axis points towards the right and spans the image columns and the $y$-axis points downward and spans the image rows.

Let $\mathbf{R}$ denote the 3D rotation that rotates the coordinate axes of the standard coordinate system to align with the same axes of a second camera having arbitrary pose. Similarly, Let $\mathbf{t}$ denote the 3D translation vector describing the position of the focal point of a second camera having arbitrary pose. Using this notation, the depth transformation can be accomplished in the following three steps:

1) Back-project $\mathbf{d}(x, y)$ to create an $(X, Y, Z)$ point cloud (as described in $\S$ II-B1),

2) Transform, i.e., rotate and translate, each point $\mathbf{p}_{i}=$ $[X, Y, Z]^{t}$ in the point cloud to generate a new point $\mathbf{p}_{i}^{\prime}=\left[X^{\prime}, Y^{\prime}, Z^{\prime}\right]^{t}$ that lies in a standard optical coordinate system centered on the second camera's focal point and having orientation that aligns with corresponding $x, y, z$-axes using equation (6),

$$
\mathbf{p}_{i}^{\prime}=\mathbf{R}\left(\mathbf{p}_{i}-\mathbf{t}\right)
$$

3) Project the $(X, Y, Z)$ point cloud using the pinhole camera equations to compute the new depth image $\mathbf{d}^{\prime}(x, y)=Z$ using equation 7 . 


$$
\begin{array}{lll}
x & = & f_{x}\left(\frac{X^{\prime}}{Z^{\prime}}\right)-\delta_{x}+c_{x} \\
y & = & f_{y}\left(\frac{Y^{\prime}}{Z^{\prime}}\right)-\delta_{y}+c_{y} \\
Z & = & Z^{\prime}
\end{array}
$$

Typically, the projected point cloud measurements fall at non-integer locations in the new depth image and the values of $\mathbf{d}^{\prime}(x, y)$ must then be interpolated using some interpolation scheme such as nearest neighbor or bilinear interpolation.

\section{RGBD Measurement Noise}

The proposed fusion algorithm relies on experimental studies of accuracy and noise for RGBD sensor measurement, e.g., the Kinect sensor. Research in [19] shows that a Gaussian noise model provides a good fit to observed measurement errors on planar targets where the distribution parameters are mean 0 and standard deviation $\sigma_{Z}=\frac{m}{2 f_{x} b} Z^{2}$ for depth measurements where $\frac{m}{f_{x} b}=-2.85 e^{-3}$ is the linearized slope for the normalized disparity empirically found in [19]. Since the 3D coordinates for $(X, Y)$ are a function of both the pixel location and the depth, their distributions are shown below:

$$
\begin{aligned}
& \sigma_{X}=\frac{x_{i m}-c_{x}+\delta_{x}}{f_{x}} \sigma_{Z}=\frac{x_{i m}-c_{x}+\delta_{x}}{f_{x}}\left(1.425 e^{-3}\right) Z^{2} \\
& \sigma_{Y}=\frac{y_{i m}-c_{y}+\delta_{y}}{f_{y}} \sigma_{Z}=\frac{y_{i m}-c_{y}+\delta_{y}}{f_{y}}\left(1.425 e^{-3}\right) Z^{2} \\
& \sigma_{Z}=\quad \frac{m}{f_{x} b} Z^{2} \sigma_{d^{\prime}}=\left(1.425 e^{-3}\right) Z^{2}
\end{aligned}
$$

These equations indicate that $3 \mathrm{D}$ coordinate measurement uncertainty increases as a quadratic function of the depth for all three coordinate values. However, the quadratic coefficient for the $(X, Y)$ coordinate standard deviation is at most half that in the depth direction, i.e., $\left(\sigma_{X}, \sigma_{Y}\right) \approx 0.5 \sigma_{Z}$ at the image periphery where $\frac{x-c_{x}}{f} \approx 0.5$, and this value is significantly smaller for pixels close to the optical axis. Variances derived from this noise model can then be used to fuse depth measurements resulting from structured light sensors with depth measurements from other sources.

\section{Methodology}

The proposed fusion approach applies the semi-dense monocular reconstruction approach referred to as Large Scale Direct (LSD) SLAM [16]. The LSD-SLAM algorithm solves the SfM problem using a direct method to compute pixel correspondences and a semi-dense method for $3 \mathrm{D}$ reconstruction. We select this approach as it does not require or impose any prior knowledge about the scene structure as required by dense reconstruction methods and it gives more 3D estimates than sparse approaches while having similar computational cost.

The LSD-SLAM SfM algorithm consists of the following three components:

1) A tracking component that estimates the pose of the camera

2) A depth map estimation component that estimates semidense depth images for keyframes

3) A map optimization component that seeks to create a 3D map of the environment that is self-consistent.

This work utilizes the first two components to explore fusion of RGBD depth images with SfM depth images. For this work, the map optimization component (3) is not used. Figure 3 depicts an overview of the proposed depth fusion algorithm.

\section{A. Time and Spatial Sampling Issues}

As mentioned previously, RGBD sensors measure depth at a rate of 30 frames per second (fps) and LSD-SLAM computes depth images only for keyframes which is a sparse subset of the measured RGB frames. Further, keyframes are not generated uniformly in time but created when the SfM algorithm detects criteria required to create a new keyframe. This condition is triggered when the current camera pose is too far from the most recent keyframe camera pose and when the current frame tracking result is "good" in the sense that the image warping correspondence objective function suggests an accurate or lowerror result. As a result, SfM-estimated depths exist only for those RGB images designated as keyframes.

Further, the spatial distribution of SfM-estimated depths within SfM keyframes are localized to only those pixels having "good" 3D reconstruction characteristics. In this sense, the quality of the depth estimate depends on accurately matching pixels along epipolar lines inscribed in the image. The matching performance here is best when there is a significant change in the image intensities along the epipolar line. Hence, 3D depth reconstruction is limited to those pixels that lie at sharp intensity changes, i.e., "edge" pixels, and further limited to those "edge" pixels that lie on edges that are roughly perpendicular to the direction of the epipolar line (see [6] for details).

The LSD-SLAM algorithm estimates depth at "good" pixel positions as a 1-dimensional Gaussian distribution specified as a mean image $\mu_{S f M}(x, y)$, i.e., the estimated depth image, and a variance image $\sigma_{S f M}^{2}(x, y)$ such that the RGB keyframe pixel at location $\mathbf{I}(x, y)$ is estimated to have depth $\mu_{S f M}(x, y)$ with uncertainties given by $\sigma_{S f M}^{2}(x, y)$. In this sense, the keyframe image $\mathbf{I}(x, y)$ augmented with the estimated depth image $\mu_{S f M}(x, y)$ is analogous in format to sensed RGBD image data. Yet, the uncertainties for the image $\mu_{S f M}(x, y)$ are given by the image $\sigma_{S f M}^{2}(x, y)$ rather than the experimentally validated uncertainties discussed in $\S$ II-C

\section{B. Image Registration Issues}

Fusing depth measurements requires knowledge of the correspondence between the depth measurements generated from the RGBD sensor and the SfM algorithm. For SfM keyframes this correspondence is trivial due to the fact that RGBD sensors support hardware registration. Hardware registration co-locates the RGBD depth image measurements, $\mathbf{d}_{r g b d}(x, y)$, and RGB appearance values, $\mathbf{I}(x, y)$. Hence, for hardwareregistered RGBD depth images, $\mathbf{d}_{r g b d}(x, y)$ is the measured depth of the surface having RGB pixel $\mathbf{I}(x, y)$. Similarly, SfMestimated depths for an RGB keyframe, $\mu_{S f M}(x, y)$, are the depths for the surface having RGB pixel $\mathbf{I}(x, y)$. Hence fusion is accomplished by fusing the measurements at corresponding $(x, y)$ locations in the RGBD depth image, $\mathbf{d}_{r g b d}(x, y)$, and the SfM depth image, $\mu_{S f M}(x, y)$.

Depth correspondences for RGB images that are not SfM keyframes must be computed from one or more SfM keyframe depth images. This article uses the most recent, i.e., closestin-time, keyframe to generate co-registered SfM depth images for arbitrary RGB images. To do so, the depth image from the 


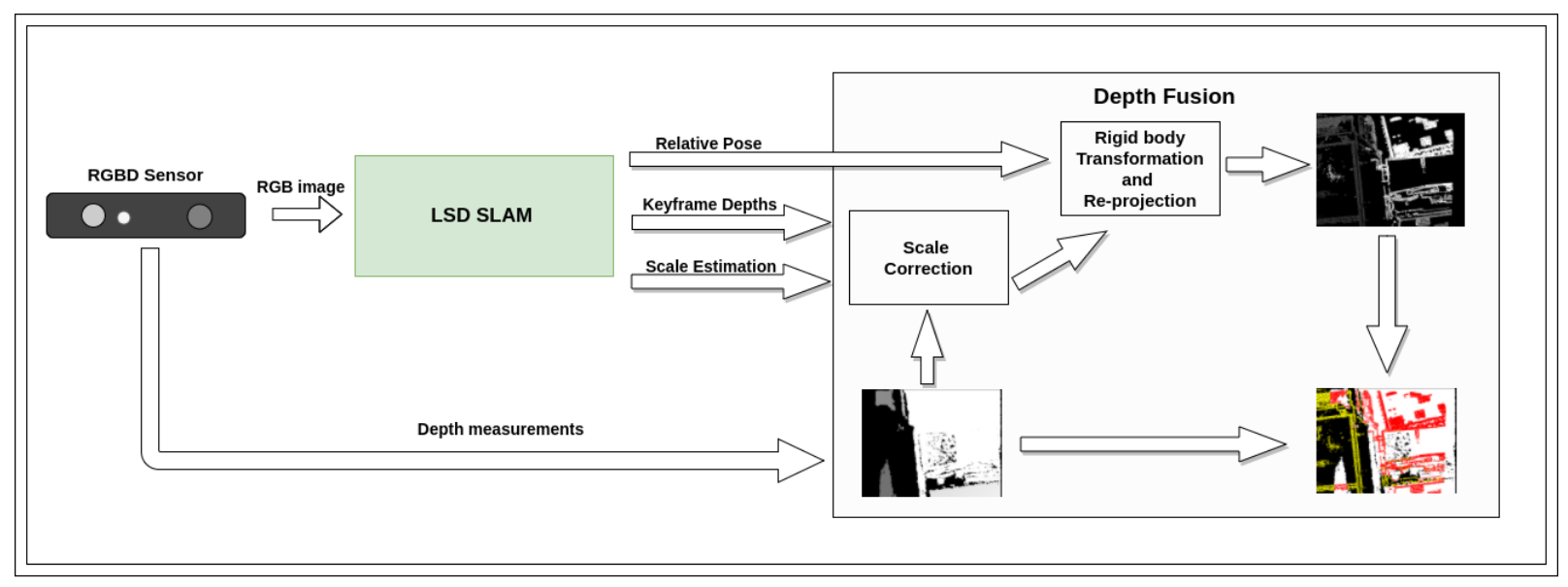

Figure 3: An overview of the proposed depth fusion algorithm.

most recent keyframe, $\mu_{S f M}(x, y)$, is back-projected II-B1 to create a 3D point cloud of SfM measurements. Using the estimated keyframe-to-camera pose change, the 3D measurements are transformed, then projected II-B2 into the RGB camera image plane using the $3 \mathrm{D}$ projection equations for the camera provided via camera calibration. The resulting depth image, $\widetilde{\mu}_{S f M}(x, y)$ is then co-registered with the RGBD depth image $\mathbf{d}_{r g b d}(x, y)$ and RGB image $\mathbf{I}(x, y)$.

Using these techniques, co-registered SfM depth images can be computed for a general RGBD image. When RGBD images correspond to SfM keyframes the registration is "automatic," i.e., no computation is necessary. In all other cases, a coregistered SfM depth image must be computed by reconstructing a 3D point cloud from a keyframe and then projecting the point cloud into the target RGB camera image using the estimated camera calibration and keyframe-to-camera-frame relative pose parameters.

\section{Resolving the Unknown SfM Scale}

The methods described in previous sections detail how coregistered SfM depth measurements are computed for every sensed RGBD frame. However, as discussed in previously, SfM depth images intrinsically have an unknown scale, $\alpha$, which reflects the fact that the solution for the scene structure is not geometrically unique, i.e., the same scene structure can be observed at a infinite number of distinct scales. Therefore fusion requires the scale of the SfM depth image to fit the scale of the real-world scene measured by the RGBD camera.

Given that the depth measurements for the RGBD depth image are co-registered with the SfM estimated depth image the scale parameter can be directly estimated by minimizing the sum of the squared depth errors [20] between the SfM depth image and the RGBD depth image. Let $V$ denote the set of $(x, y)$ positions that have valid depth measurements for "standard" fusion as described in the section III-D, Equation (9) shows the error function used to compute the unknown scale value and equation (10) shows the solution $\widehat{\alpha}$ that minimizes this error.

$$
\begin{gathered}
e(\alpha)=\sum_{(x, y) \epsilon V}\left\|\mathbf{d}_{r g b d}(x, y)-\alpha \mu_{S f M}(x, y)\right\|^{2} \\
\widehat{\alpha}=\sum_{(x, y) \epsilon V} \frac{\mathbf{d}_{r g b d}(x, y)}{\mu_{S f M}(x, y)}
\end{gathered}
$$

\section{RGBD and SfM Depth Fusion}

For fusing measurements we consider the structured-light measurement of the RGBD sensor to generate a distribution for the unknown true depth of the scene surfaces at each $(x, y)$ pixel in the depth image. These measurements are considered to be independent and identically distributed to the measurements of the true unknown depth of the scene surfaces from the registered SfM estimated depths. With these assumptions, solving the depth fusion problem is equivalent to estimating the posterior distribution of the true scene depth at each $(x, y)$ position given the distributions for the RGBD and SfM depth values.

Fortunately, previous sections show that Gaussian models are appropriate distributions for both the RGBD and SfM depth values and the parameters of these models are either known (see $\S$ III-C) or estimated continuously (see $\S$ III-A). When both distributions are Gaussian, the posterior distribution can be found analytically and is a well-known result used in pattern recognition and other prediction frameworks, e.g., the Kalman filter as discussed in [21]. Specifically, let the Gaussian noise for RGBD depth at position $(x, y)$ be represented as $\mathcal{N}\left(\mathbf{d}_{r g b d}, \sigma_{r g b d}^{2}\right)$ and the Gaussian noise for the co-registered SfM depth image at position $(x, y)$ be $\mathcal{N}\left(\mu_{S f M}, \sigma_{S f M}^{2}\right)$. The posterior distribution on the unknown true depth at position $(x, y)$ is also Gaussian and let $\mathcal{N}\left(\mu_{\text {fused }}, \sigma_{\text {fused }}^{2}\right)$ denote the mean and variance parameters of this distribution. Equations (11) and (12) provide optimal estimates of the mean and variance of the fused depth at position $(x, y)$. The best estimate of the fused depth is given by the highest probability value in the posterior distribution which is the mean fused image, $\mu_{\text {fused }}(x, y)$. 


\begin{tabular}{|c|c|c|c|}
\hline Experiment & $\begin{array}{c}\text { RGBD-only depths } \\
(\%)\end{array}$ & $\begin{array}{c}\text { SfM-only depths } \\
(\%)\end{array}$ & $\begin{array}{c}\text { Fused depths } \\
(\%)\end{array}$ \\
\hline \hline 1 & 67.7 & 6.7 & 25.5 \\
\hline 2 & 55.5 & 10.0 & 34.3 \\
\hline 3 & 68.1 & 15.1 & 16.6 \\
\hline \hline Average & 63.3 & 10.3 & 26.3 \\
\hline
\end{tabular}

Table I: Ratios of depth measurements to total depths

$$
\begin{gathered}
\mu_{f u s e d}=\frac{\mathbf{d}_{r g b d} \sigma_{S f M}^{2}+\mu_{S f M} \sigma_{r g b d}^{2}}{\sigma_{S f M}^{2}+\sigma_{r g b d}^{2}} \\
\sigma_{\text {fused }}^{2}=\frac{\sigma_{r g b d}^{2} \sigma_{S f M}^{2}}{\sigma_{r g b d}^{2}+\sigma_{S f M}^{2}}
\end{gathered}
$$

\section{Results}

We have applied the depth fusion algorithm to sets of RGBD images of a variety of indoor scenes. RGBD image sequences were recorded using an Orbbec Astra RGBD sensor. The LSD-SLAM algorithm was applied to the RGB image sequence using the factory provided intrinsic camera calibration parameters. Each sequence included approximately 60 seconds of RGBD image data at the rate of $30 \mathrm{fps}$. Experimentally recorded RGB images were processed offline by the LSD-SLAM algorithm to generate SfM depth images. Experiments initialize the LSD-SLAM algorithm with the first recorded depth image from the RGBD sensor to facilitate the initial scale approximation. The output from the LSD-SLAM algorithm consisting of the relative pose for every tracked frame and the depth map for each keyframe was then recorded. The fusion algorithm was then applied using the recorded RGBD image stream and LSD-SLAM output files to generate the results shown in this section.

Experiment 1 depicts an indoor office scene at the university. This scene includes specular and dark surface structures at close range that are not measured by the RGBD sensor. Yet, the SfM algorithm estimates depths at a number of locations (on the podium) where there are significant intensity changes. These additional depths are evident in the fused results, which includes SfM-only depth measurements in regions in the vicinity of image edges. The experiment demonstrates that depth fusion can improve depth images by obtaining depths from surfaces not measurable by the RGBD sensor.

Experiment 2 depicts a hallway in the UNCC EPIC building that includes both specular surfaces and high intensity illumination from overhead lights. The experiment is a second example showing that depth fusion bolsters over all depth measurement performance by providing the depths when RGBD sensors fail. The SfM takes advantage of the patterns on the surface and estimates the depths irrespective of the nature of its reflectance properties and color.

Experiment 3 depicts the UNCC faculty conference hall. Here a number of scene structures lie beyond the measurement range of the RGBD sensor. Yet, the SfM algorithm is able to estimate the depth of these scene structures (albeit at high variance) providing depths that would otherwise not be possible.
Table I quantifies the amount of additional depth information provided via RGBD-SfM depth image fusion. On average, approximately $63 \%$ of the fused depths originate from the RGBD sensor alone, approximately $10 \%$ original from the SfM algorithm alone, and approximately $26 \%$ of the fused depths results from RGBD-SfM fusion. The addition of $10 \%$ of novel depth data is a significant contribution. Further, fused depths account for roughly $26 \%$ of depth image data and the measurement error for all of these measurements will be reduced by the fusion. Variance reduction will be greatest for low-variance SfM depth estimates which are typically in textured scene locations close to the camera. Yet, we note that by inspection of equation (12), it is theoretically impossible for the variance of any fusion result to increase.

\section{Conclusions}

The SfM depth estimation complements the RGBD sensor measurements and can provide depths when RGBD sensors fail. The proposed depth fusion algorithm provides an effective way to augment the RGBD depth stream and results in improved depth images. The experiments conducted show improvements in the resulting depth images for a number of scenarios where structured light depth estimation fails. This includes successfully capturing depth for out-of-range RGBD depth locations and successfully capturing depth measurements from specular and dark objects.

In future work, the proposed depth fusion method can be generalized to address depth image locations that include measurements having non-Gaussian noise distributions. Also, experimental results show that the scale and trajectory estimation of SfM depths are not always accurate. Inclusion of RGBD-SfM fused depth data into the SfM algorithm can be exploited to improve the camera pose estimation and increase reconstruction accuracy.

\section{REFERENCES}

[1] K. Litomisky, "Consumer rgb-d cameras and their applications," 2012.

[2] Z. Zhang, "Microsoft kinect sensor and its effect," IEEE MultiMedia, vol. 19, pp. 4-10, Apr. 2012.

[3] C. Jing, J. Potgieter, F. Noble, and R. Wang, "A comparison and analysis of rgb-d cameras' depth performance for robotics application," in 2017 24th International Conference on Mechatronics and Machine Vision in Practice (M2VIP), pp. 1-6, Nov 2017.

[4] A. Kadambi, A. Bhandari, and R. Raskar, 3D Depth Cameras in Vision: Benefits and Limitations of the Hardware, pp. 3-26. Cham: Springer International Publishing, 2014.

[5] Z. Zhang, "A flexible new technique for camera calibration," IEEE Trans. Pattern Anal. Mach. Intell., vol. 22, pp. 1330-1334, Nov. 2000.

[6] J. Engel, J. Sturm, and D. Cremers, "Semi-dense visual odometry for a monocular camera," in 2013 IEEE International Conference on Computer Vision, pp. 1449-1456, Dec 2013.

[7] B. Triggs, P. F. McLauchlan, R. I. Hartley, and A. W. Fitzgibbon, "Bundle adjustment - a modern synthesis," in Proceedings of the International Workshop on Vision Algorithms: Theory and Practice, ICCV '99, (London, UK, UK), pp. 298-372, Springer-Verlag, 2000.

[8] Y. M. Wang, Y. Li, and J. B. Zheng, "A camera calibration technique based on opencv," in The 3rd International Conference on Information Sciences and Interaction Sciences, pp. 403-406, June 2010.

[9] J. Engel, V. Koltun, and D. Cremers, "Direct sparse odometry," IEEE Transactions on Pattern Analysis and Machine Intelligence, vol. 40, pp. 611-625, March 2018.

[10] C. Harris and M. Stephens, "A combined corner and edge detector," in In Proc. of Fourth Alvey Vision Conference, pp. 147-151, 1988. 

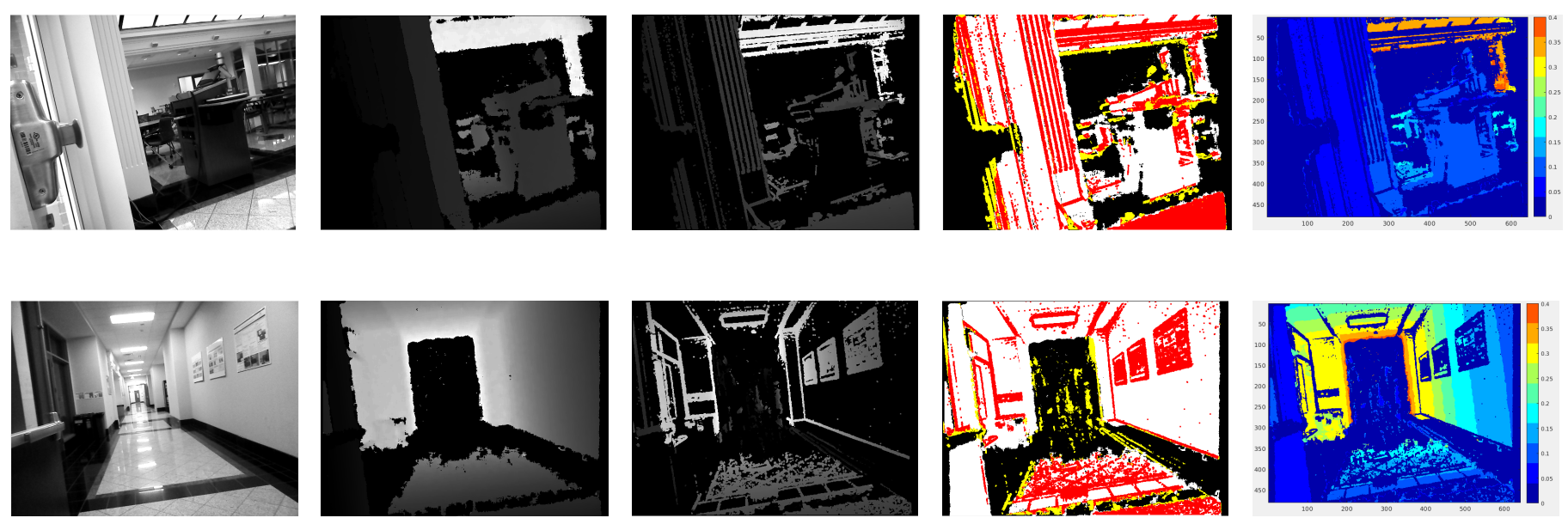

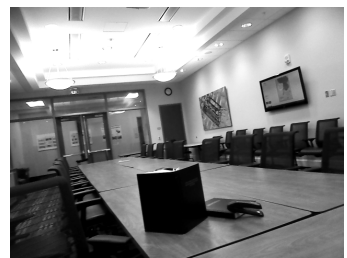

(a)

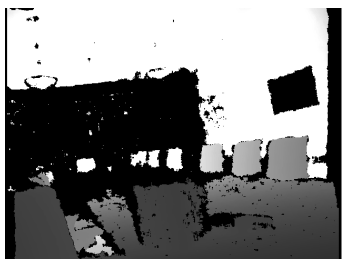

(b)

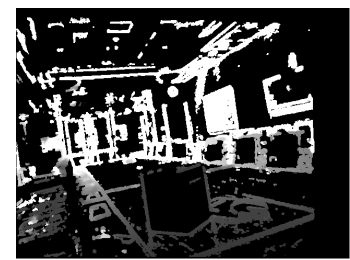

(c)

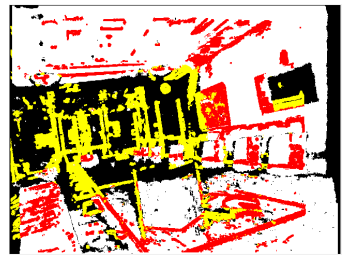

(d)

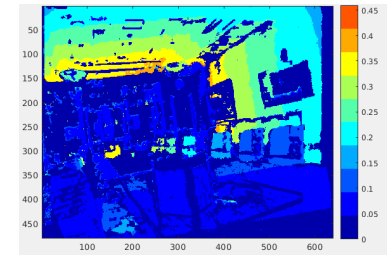

(e)

Figure 4: Results for three experiments are shown. Images shown are organized into separate columns. Column (a) shows a grayscale image of the scene (b) shows the sensed RGBD depth image (c) shows the SfM-estimated depth image (d) shows the fused image and (e) shows the standard deviation for fused depths (in $m$.). The fused image has been color-coded as follows: (white) denotes depth locations sensed only by the RGBD sensor, (yellow) denotes depth locations only sensed via SfM, (red) denotes fused (RGBD+SfM) depth locations and (black) denotes depth locations without RGBD or SfM measurements.

[11] D. G. Lowe, "Distinctive image features from scale-invariant keypoints," International Journal of Computer Vision, vol. 60, pp. 91-110, Nov 2004.

[12] S. Baker and I. Matthews, "Lucas-kanade 20 years on: A unifying framework," Int. J. Comput. Vision, vol. 56, pp. 221-255, Feb. 2004.

[13] G. Klein and D. Murray, "Parallel tracking and mapping for small AR workspaces," in Proc. Sixth IEEE and ACM International Symposium on Mixed and Augmented Reality (ISMAR'07), (Nara, Japan), November 2007.

[14] H. Bay, A. Ess, T. Tuytelaars, and L. Van Gool, "Speeded-up robust features (surf)," Comput. Vis. Image Underst., vol. 110, pp. 346-359, June 2008.

[15] C. Kerl, J. Sturm, and D. Cremers, "Dense visual slam for rgb-d cameras," in 2013 IEEE/RSJ International Conference on Intelligent Robots and Systems, pp. 2100-2106, Nov 2013.

[16] J. Engel, T. Schöps, and D. Cremers, "Lsd-slam: Large-scale direct monocular slam," pp. 834-849, 2014.

[17] R. KÃ Emmerle, G. Grisetti, H. Strasdat, K. Konolige, and W. Burgard, "G2o: A general framework for graph optimization," in 2011 IEEE International Conference on Robotics and Automation, pp. 3607-3613, May 2011.

[18] Y. Ma, S. Soatto, J. Kosecka, and S. S. Sastry, An Invitation to 3-D Vision: From Images to Geometric Models. SpringerVerlag, 2003.

[19] J. Papadakis and A. R. Willis, "Real-time surface fitting to rgbd sensor data," in SoutheastCon 2017, pp. 1-7, March 2017.

[20] C. M. Bishop, Pattern Recognition and Machine Learning (Information Science and Statistics). Secaucus, NJ, USA: Springer-Verlag New York, Inc., 2006.

[21] P. S. Maybeck, "Stochastics models, estimation, and control: Introduction," 1979. 